\title{
MONITORING DEFORMATIONS OF A WOODEN CHURCH TOWER BY LASER SCANNING
}

\author{
LINH TRUONG-HONG ${ }^{1 *}$, RODERIK LINDENBERGH ${ }^{1}$, PIETER WOUDENBERG $^{2}$, \\ WOLFGANG GARD ${ }^{2}$, JAN-WILLEM VAN DE KUILEN KI $^{2,3}$ \\ ${ }^{1}$ Department of Geoscience and Remote Sensing, Faculty of Civil Engineering and Geosciences \\ ${ }^{2}$ Department of Engineering Structures, Faculty of Civil Engineering and Geosciences \\ Delft University of Technology \\ Stevinweg 1, 2628 CN Delft, The Netherlands \\ ${ }^{3}$ Chair of Wood Technology, Technical University of Munich \\ Arcisstraße 21, 80333 München, Germany \\ e-mail: 1.truong@tudelft.nl (*corresponding author) \\ email: \{r.c.lindenbergh,w.f.gard,j.w.g.vandekuilen\} -@tudelft.nl \\ email: pieter.woudenberg@hotmail.com
}

Keywords: Point Cloud, Deformation, Monitoring, Ransac, Segmentation, Heritage Structure, Wooden church tower

\begin{abstract}
Churches are part of heritage structures that take an important role in Europe's cultural identity. As such, these structures must be protected to prevent catastrophic collapse and any damage must be reported timely to establish planning to maintenance and restoration. This can be achievable when the churches are monitored periodically with regular intervals. However, this monitoring strategy has not been available in most of the Europe's churches for a number of reasons, complexity of the structures and limited budget are just two of them. Laser scanning has been widely used in capturing rich three-dimensional (3D) topographic data of visible surfaces of a structure with high accuracy. This paper presents a methodology to determine the shape and possible deviation from verticality of the church's tower for monitoring deformation using a terrestrial laser scanner. The 500-year old wooden tower of St. Bavo Church in Haarlem, Netherlands is selected as a case study. First, point clouds of the tower captured from different views are registered into the same coordinate system. Second, a RANSAC method is employed to extract point clouds of a whole façades of the tower. Next, a point and surface-based method is proposed to compute the deformation of the surface from its data points. The results indicate that there is slightly different deformation between the tower facades in the same story and in neighbour stories. Moreover, the maximum total relative deformation at Story 7 of the tower by $0.63 \mathrm{~m}$.
\end{abstract}

\section{INTRODUCTION}

Monitoring of structures is becoming an important task allowing to discover deficiencies, movements, rotations and any time related effects, allowing to better protect the structures and to predict and prevent possible adverse events. Common methods are based on measured signals from sensors (e.g. accelerometers, deformometers) or on measured discrete points by using 
digital levelling and total stations. However, for heritage structures, non-contact methods are preferable because of cultural value restrictions, that prohibit any structural alterations. By using traditional surveying equipment (e.g. digital levelling, total station), resulting deformations of structures are based on a point-wise approach. This requires high accuracy of the measuring devices. However, with such methods, as often only a few points on the structure can be measured, the deformation may not fully reflect the actual condition of the structure. As such, assessment results must only be interpreted by experienced inspectors.

Laser scanning has appeared as one of the best available technologies to capture details of structures' surfaces quickly, efficiently and accurately. The accuracy of a single point that can be achieved is about a millimetre, whereas it can be up to a centimetre for registered points coming from multiple scanning stations. Laser scanning has been widely used in various applications, e.g. finite element mesh generation [1], structural assessment [2, 3] and deformations [4]. In terms of using laser scanning for deformation measurements, the accuracy of registered points may not fully satisfy the requirements, but surface-wise methods are often used to determine the surface deformation based on a point cloud of the surface. Such approaches can compensate the accuracy of the point cloud by exploring correlations between the points, which allows to define an extent of a (surface) deformation or a surface anomaly in an accurate and reliable way [5]. In this paper, a methodology is presented to measure deformations of the wooden tower of St. Bavo Church in Haarlem, the Netherlands, by using terrestrial laser scanning data. Main challenge of this study is that the long-term behaviour of the wooden tower has resulted in non-uniform deformations in the entire structure, which requires to determine tower's geometry is massive point clouds

2 RELATED WORK
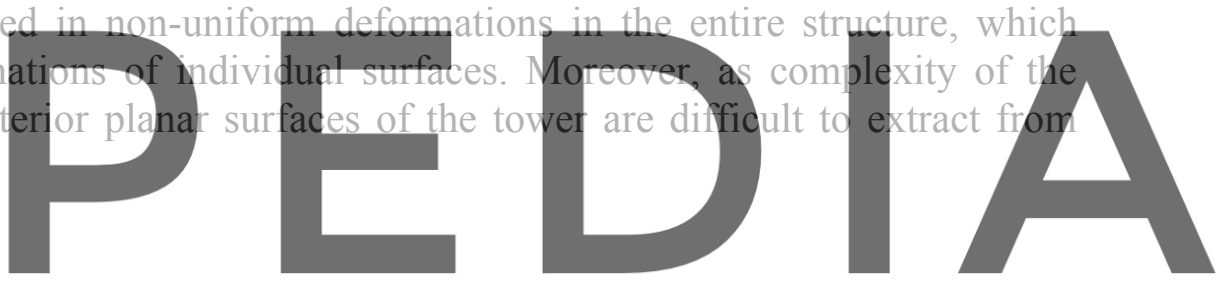

Laser scanning has been commonly used to capture surfaces of structural components for

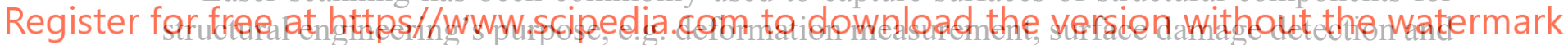

finite element generation. A systematic overview of a wide range of applications of laser scanning in heritage and civil structures was recently published at [6]. This survey is restricted to deformation measurements of the heritage structures.

González-Aguilera el at. [7] measure deformation of the Mediaeval Wall of Avila (Spain) by analysing a point cloud of the wall acquired by a Trimble GX200 laser scanner. Surfaces of the wall are extracted by using a planar surface fitting and then multiple vertical and horizontal cross-sections of the fitted surfaces are used to determine wall deformation between different epochs. Also using a surface-based method, in measuring deformation of the Garisenda and Asinelli towers in Bologna (Italy), Pesci et al. [8] propose two reference surfaces, which are (i) the vertical surface through the base of the towers, and (ii) the best fitting plane of a point cloud of a surface. Deformation is herein the distance from the points to the reference points. Additionally, Bonali et al. [9] determine deformation of the Cantalovo church (Italy) as distances from point clouds to surfaces to its fitting primitive. 
In measuring deformations of the Asinelli tower in Bologna, Bertacchini et al. [10] slices the point cloud of the tower in the elevation direction at an interval $10 \mathrm{~cm}$ and computes the centroid of each horizontal slice, which is considered as the point of a barycentre axis for determining deviation from verticality (out-of-plumb) of the tower [10]. Similarly, Bhadrakom and Chaiyasarn [11] reconstruct the central line of a tower of the Ayutthaya temples (Thailand) by measuring a tilt angle. A point cloud of the tower is separated in multiple horizontal slices along elevation. As the tower shape is a cylinder, the center points of the horizontal slice are determined as the centre of a fitting cylinder. The strategy using a central line to determine a deformation is also applied by Muszynski and Milczarek [12] when they measure the inclination of chimney's axis.

Although those methods can determine deformations of historic structures, they still have some drawbacks. First, methods-based fitting surface through all points of the façade may not be applicable for structures subjected to non-linear or multi-linear deformations along the elevation of the façade. Moreover, using the best fitting surface as the reference surface may include an error of data acquisition and registration, and existing damage in the surface, which lead to relative low accuracy of the reference. Second, using a point cloud of horizontal slices to determine the central line is applicable only when the data points cover major surfaces of the section. In addition, when a small damage is available on the cross-section, a change of the centre point compared to one from an undamaged cross-section may not be detected because the centre point determination is dominated by a large amount of the points on an undamaged area. Moreover, when t not perpendicular to horizontal slice is slight
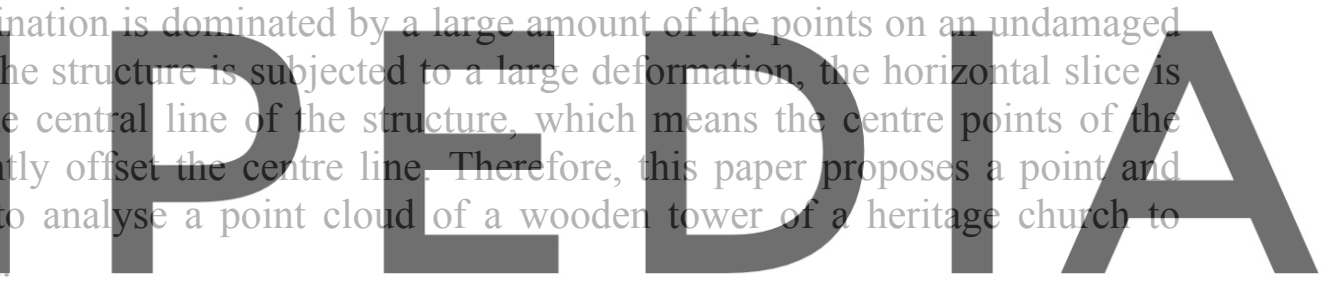

Register for free at https//www.scipedia.com to download the version without the watermark 

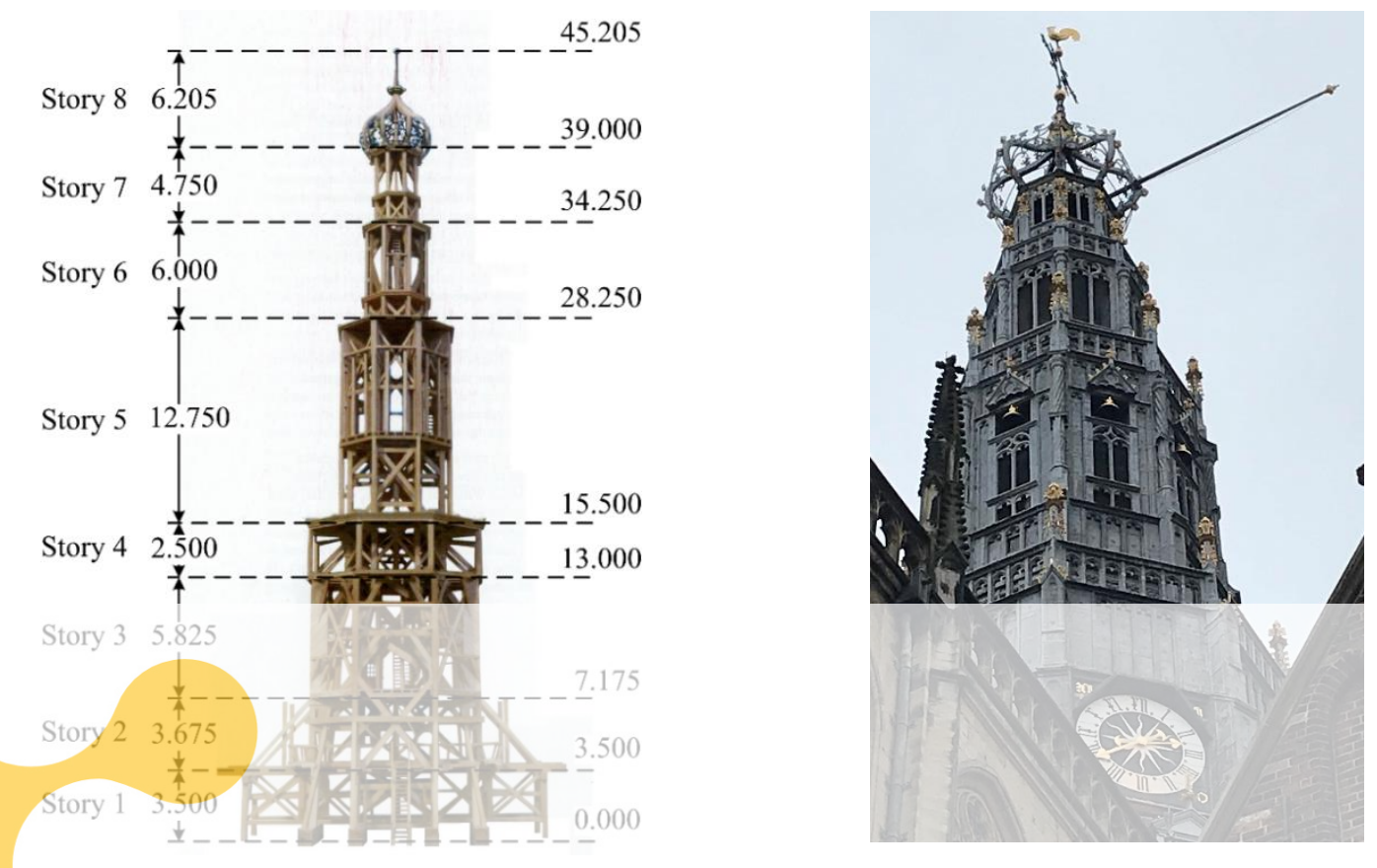

a) A 1:25 scale of the timber frame

b) A photo of the tower
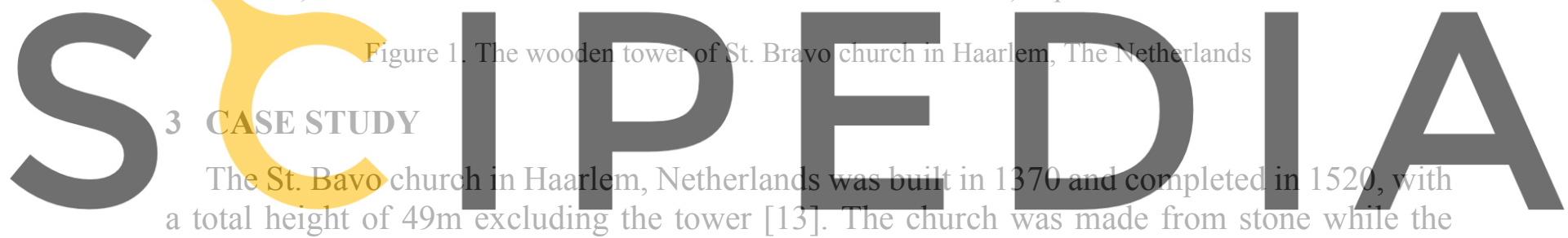

European Oakwood was used to build the current tower, which exterior surfaces of the tower

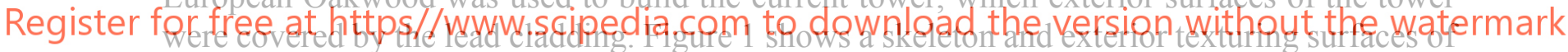

the wooden tower. The skeleton model was made based on hand measurements.

\section{PROPOSED METHODS}

A goal of this study is to determine the shape and possible deviation from verticality of the wooden tower of the St. Bavo Church, which is then used for monitoring its deformation. The proposed method consists of 3 steps as shown in Fig. 2. Step 1 and 2 extract point clouds of surfaces representing octagon facades of the tower, which are done using as-built tools in software, while the proposed automatic algorithm is developed to compute deformation of each surface of the tower (Step 3). 
Step 1: Data acquisition

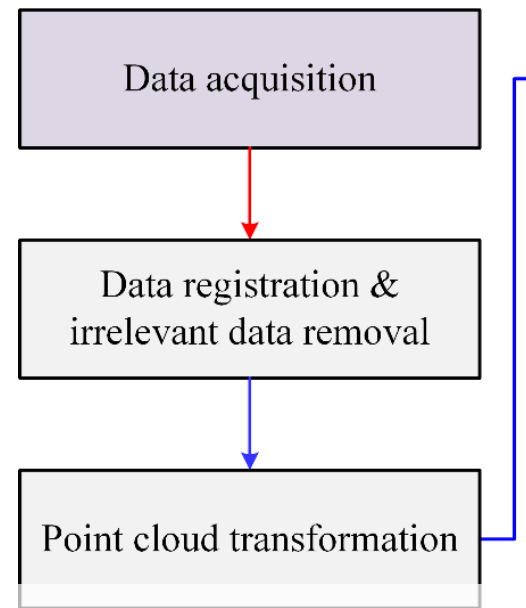

Step 2: Segmentation

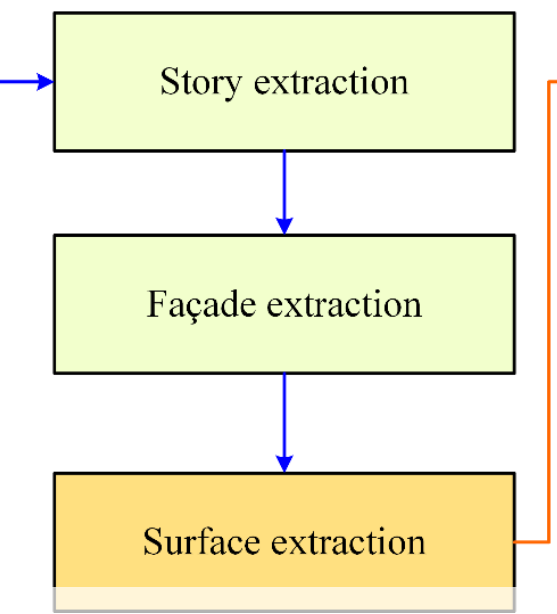

Step 3: Deformation

Estimate undeformed \& deformed surface:

$\mathrm{S}_{0}=\left\{\mathrm{P}_{0}, \mathrm{n}_{0}\right\} \& \mathrm{~S}_{1}=\left\{\mathrm{P}_{1}, \mathrm{n}_{1}\right\}$

Compute an inclination slope, $\Delta_{\text {slope }}$

Compute cumulative relative deformation, $\delta_{\text {cumrel }}$

Figure 2. Proposed workflow for deformation measurement

\subsection{Data acquisition}

Geometric data of exterior surfaces of the tower are acquired by a Leica ScanStation P40 on Feb. 2019. This scanner was selected because it can capture visible surfaces at high details quickly and accurately from a long distance. A Leica ScanStation P40 has a maximum scanning

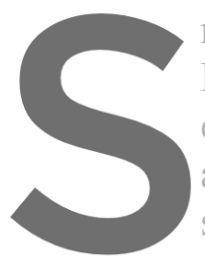

range at $270 \mathrm{~m}$ and an an

However, due to the com

exterior surfaces of the

a sampling step of $0.8 \mathrm{~m}$

step of $6.2 \mathrm{~mm}$ on the su

A total of 10 scanning stations was established to
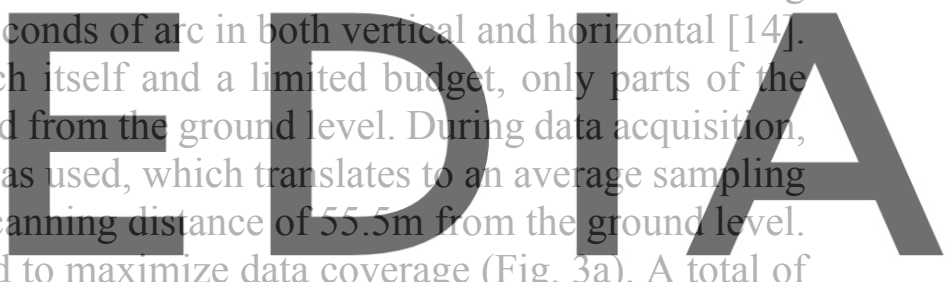

57.64 million acquired points represent the tower.

Register for free at https//WwW.scipedia.com to download the version without the watermark

To register point clouds from 10 scanning stations, 4.5inches circular black-white artificial

targets were used as mutual references between 2 scanning stations. The registration process

was done within the Leica Cyclone software [15] with the registration error about $5 \mathrm{~mm}$. However, during data acquisition, although only parts of the tower was selected to capture, the overlap between scans from different stations and irrelevant surfaces are often available. As such, irrelevant surfaces were manually cleaned within the Leica Cyclone software [15]. Moreover, point density is linear proportional to the scanning range, which can cause a negative impact on surface extraction because of un-even point distribution. In addition, as dense data points in overlap areas may not be necessary in determining deformations, the point cloud is down-sampled with the distance between two adjacent points of no more than $5 \mathrm{~mm}$, which also reduces intensive computation in data processing. This reduced the initial scanning data set from 57.64 million points to 22.44 million points. Finally, the point cloud in the scanning coordinate system (Fig. 3b) is translated to a tower coordinate system (Fig. 1a), which means the elevation of the story-based point cloud matches the elevation on the sketched skeleton model (Fig. 1a). 


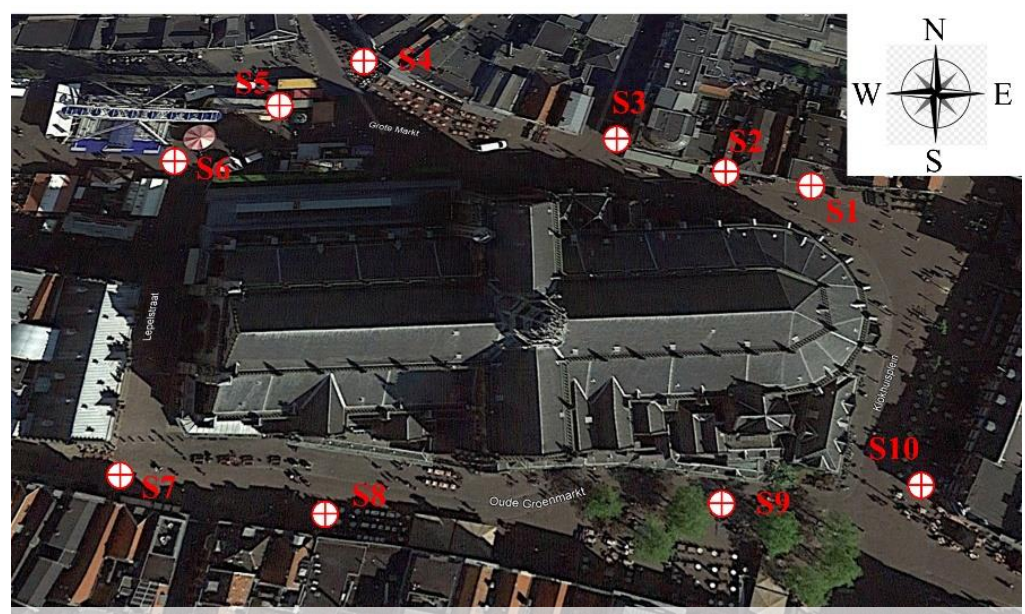

a) Locations of the scanning stations

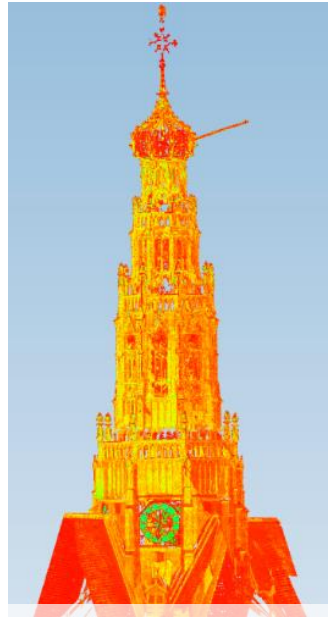

b) A point cloud of the tower

Figure 3. Data acquisition for the tower of the St. Bravo Church

\subsection{Segmentation}

Well-known segmentation methods for point clouds are region growing-based methods [16, 17] and Random Sample Consensus. (RANSAC) methods [18]. However, as region growing
based methods are time consuming and difficulty to sclect appropriate parameters to segment
surfaces of the complex structures like this tower, the RANSAC method efficient in extracting
planar surfaces is selected in this study. The RANSAC method starts randomly to seldct a
number of points to generate a hypothesis close to a truth geometric model, and the hypothesis
is then evaluated based on the number of inlier noints, which are the points having an error, which is commonly Euclidian distance, less than a threshold. The final estimation model is the

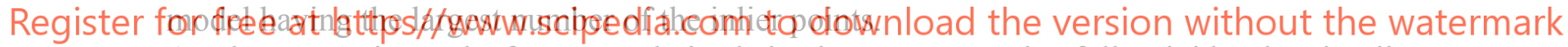
As the tower is made from wood, its behaviour may not be fully rigid. That implies a deformation of each surface/facade in each story and of each story are different. As such, surfaces on the octagon façades of each story are needed to extract. However, if the RANSAC method is applied on the data set of the tower, the desired results may not be obtained because the point clouds of different stories may impact to evaluate a local surface of a façade. Moreover, using the point cloud of the tower as a whole input data for segmentation is unnecessary since in this case the RANSAC method must process a large data set to extract the façade surfaces.

As such, the point cloud of each story is first manually extracted based on the elevations of the bottom and top of the story (Fig. 4a), and then the point cloud of each façade for each story was manually separated through the columns between the facades (Fig. 4a and b). This work was done within CloudCompare software [19]. Notably, although this work is manually, it does not affect the façade surface extraction. Finally, the RANSAC method proposed by Schnabel et al. [18], which is implemented in CloudCompare [19], is selected to extract planar surfaces. The segmentation is required to define three important input parameters: (1) the minimum number of points to define the final model - min_ptc, (2) the maximum distance from the point to the model $\varepsilon_{\max }$, and (3) the deviation of points' normal vectors to the model normal at the points - 
$\alpha$. These values are respectively pre-defined as 300 points, $0.01 \mathrm{~m}$ and 5 degrees. Although the RANSAC method can extract planar surfaces efficiently, a resulting segment may contain several objects' surfaces, so-called spurious surfaces. This issue can be eliminated by implementing a connected component labelling [20] to cluster the points of the segment, in which the distance between the points in the cluster is no larger than $0.2 \mathrm{~m}$. The final resulted segments for each façade for deformation measurement are shown in Fig. 4c.

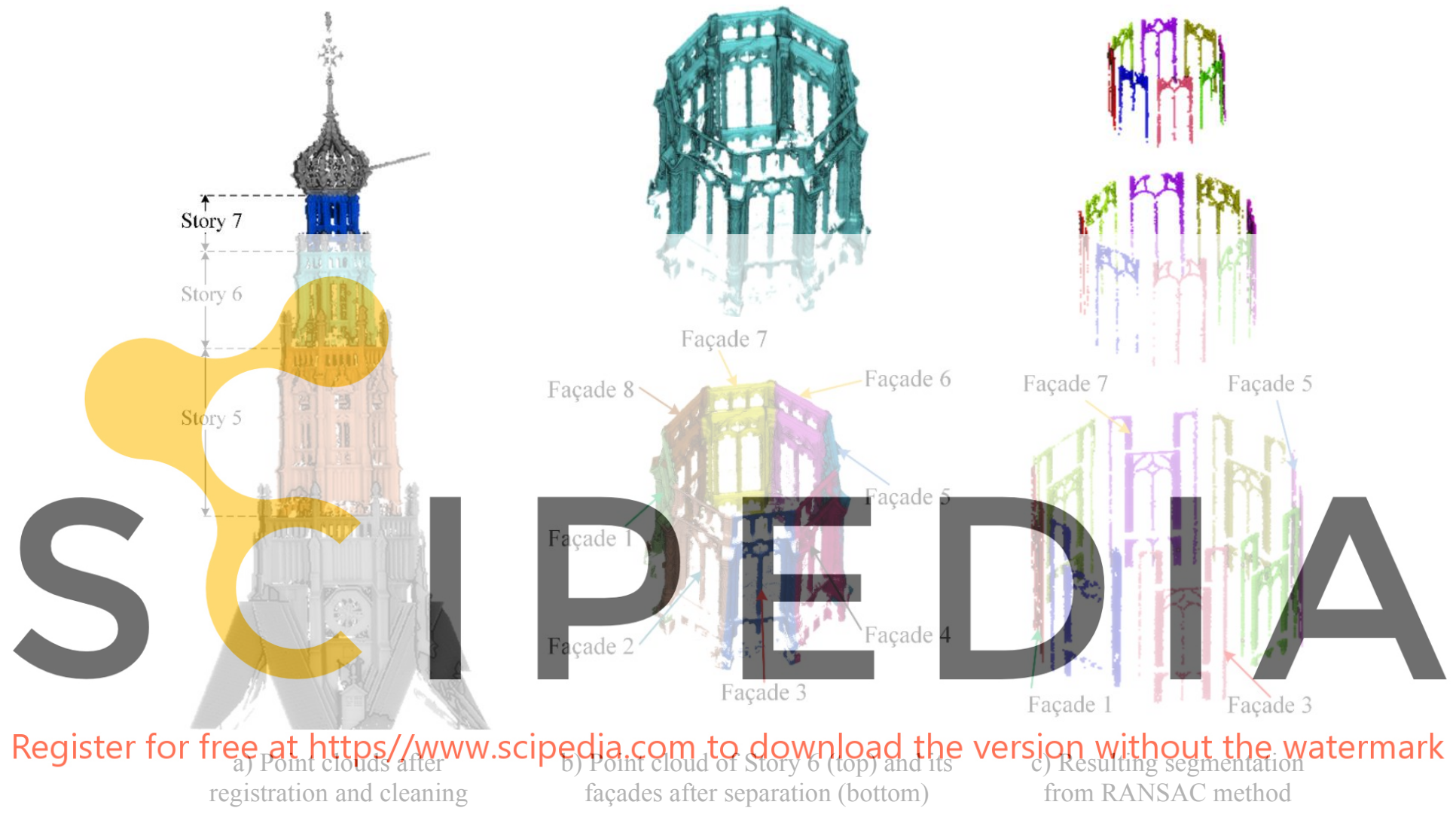

Figure 4. Extracting a point cloud of facades' surfaces

\subsection{Deformation measurement}

As the tower is made from wood, a deformation of each surface/facade in each story and of each story can be different because connections between elements are not rigid. In this study, the deformation of the tower computed from the point cloud is based on following assumptions: (1) an un-deformed or initial surface is the perfectly vertical surface, (2) a segmented surface represents the façade surface, (3) the deformation of the tower is herein defined as the out-ofplumb of the vertical surface and (4) the deformation is a relative deformation respecting to the bottom story or the bottom of Story 5. The schematic diagram for computing relative deformation of the surface at each story is shown in Fig. 5.

From the point cloud of the segmented planar surface, a fitting surface is estimated using a principal component analysis [21], in which the normal vector of the surface is the eigen-vector $\left(\mathrm{n}_{1}=\left(\mathrm{n}_{1} . \mathrm{x}, \mathrm{n}_{1} . \mathrm{y}, \mathrm{n}_{1} . \mathrm{z}\right)\right)$ corresponding to the smallest eigen-value determined from a covariance 
matrix (Eq. 1).

$$
C=\sum_{i=1}^{N}\left(p_{i}-p_{0}\right)\left(p_{i}-p_{0}\right)^{T}
$$

where $\mathrm{p}_{0}$ is the centroid point computed from the points $\mathbf{P}=\left\{\mathbf{p}_{\mathrm{i}} \in \mathbf{R}^{3} \mid \mathrm{i}=[1, \mathrm{~N}]\right\}$
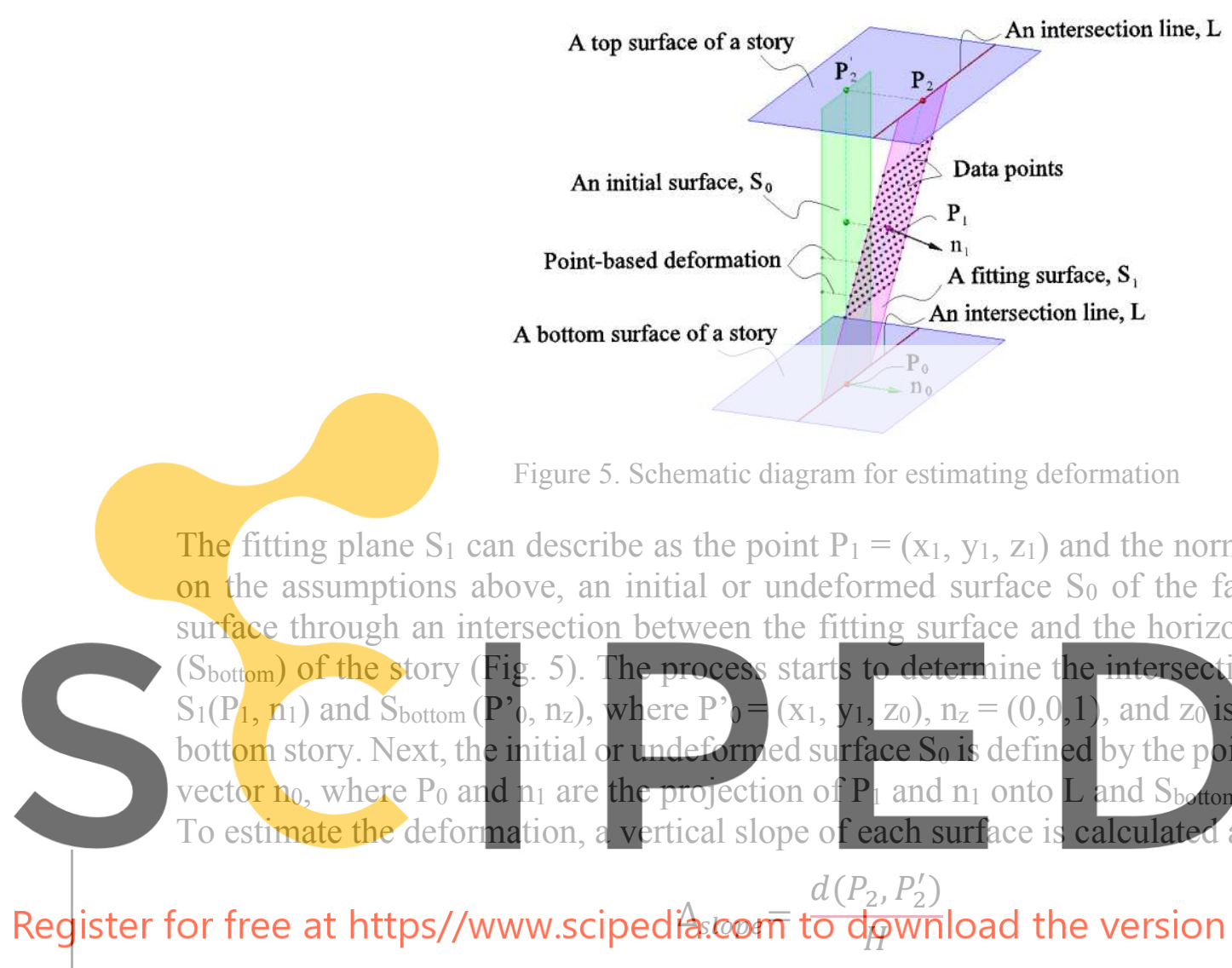

Figure 5. Schematic diagram for estimating deformation

The fitting plane $\mathrm{S}_{1}$ can describe as the point $\mathrm{P}_{1}=\left(\mathrm{x}_{1}, \mathrm{y}_{1}, \mathrm{z}_{1}\right)$ and the normal vector $\mathrm{n}_{1}$. Based on the assumptions above, an initial or undeformed surface $S_{0}$ of the façade is the vertical surface through an intersection between the fitting surface and the horizontal bottom surface (S Sottom) of the story (Fig. 5). The process starts to determine the intersection line (L) between $\mathrm{S}_{1}\left(\mathrm{P}_{1}, n_{1}\right)$ and $\mathrm{S}_{\text {bottom }}\left(\mathrm{P}^{2}\right.$ bottom story. Next, the vector no, where $P_{0}$ and To estimate the deform
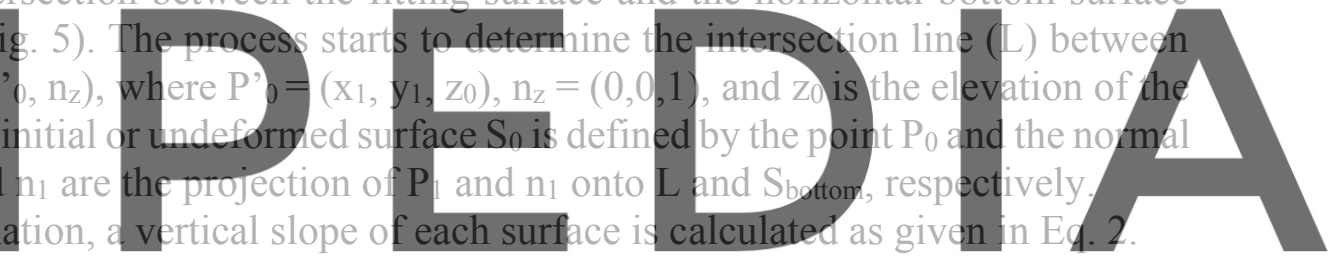
$d\left(P_{2}, P_{2}^{\prime}\right)$

where $d\left(P_{2}, P_{2}^{\prime}\right)$ is the distance from the point $P_{2}$ to $S_{0}, P_{2}$ is the projection of $P_{1}$ onto the intersection line between $\mathrm{S}_{1}$ and the horizontal top surface of the story, $\mathrm{H}$ is the height of the story.

Next, point and surface-based methods for computing a relative deformation of the surface is given as expressed in Eq. 3, which are distances from the data points of the segmented surface to the undeformed surface $\mathrm{S}_{0}\left(\mathrm{P}_{0}, \mathrm{n}_{0}\right)$.

$$
\delta_{r e l}=d\left(\boldsymbol{p}_{i}, S_{0}\right)=\frac{n_{0} \cdot x * x_{i}+n_{0} \cdot y * y_{i}+n_{0} \cdot z * z_{i}+d_{0}}{\sqrt{n_{0}^{2} \cdot \mathrm{x}+n_{0}^{2} \cdot \mathrm{y}+n_{0}^{2} \cdot \mathrm{z}}}
$$

where $\mathbf{P}=\left\{\mathbf{p}_{\mathrm{i}} \in \mathbf{R}^{\mathbf{3}} \mid \mathrm{i}=[1, \mathrm{~N}]\right\}$ are the point cloud of the façade surface and $d_{0}=n_{0} \cdot x * x_{0}+$ $n_{0} . y * y_{0}+n_{0} . z * z_{0}$. For the surface-based method, $\mathrm{p}_{\mathrm{i}}$ is the point on the fitting surface $\mathrm{S}_{1}$.

Finally, cumulative relative deformation $\left(\delta_{\text {cumrel,i }}\right)$ of Story i is calculated according to Eq. 4 .

$$
\delta_{\text {cumrel, } i}=\delta_{\text {cumrel,i-1 }}+\delta_{\text {rel, } i}
$$

where $\delta_{\text {cumrel, },-1}$ is the cumulate relative deformation-based surface at Story $\mathrm{i}-1$, and $\delta_{\text {rel, }}$ is the relative deformation from the point- or surface-based methods of Story i. 


\subsection{Results and Discussions}

As exterior surfaces of the tower are captured by laser scanning, geometric data of the tower skeleton is not available. Based on dimensions from the skeleton models and point clouds of exterior facades of the tower, in this data acquisition, only data points representing the exterior surfaces of Story 5 to Story 7 are available. Vertical slopes of facades are illustrated in Fig. 6, in which the absolute vertical slopes vary from $0.26 \%$ (Façade 2) to $3.21 \%$ (Façade 8 ) for Story 5, from $0.12 \%$ (Façade 2) to $3.66 \%$ (Façade 8) for Story 6, and $0.44 \%$ (Façade 2) to $3.73 \%$ (Façade 8) for Story 7. Even though in the same story, the vertical slopes of two opposite facades are different. For example, in Story 5, the vertical slopes of Façade 3 and 7 are respectively $-1.36 \%$ and $2.47 \%$. Moreover, the slopes of the same facades in different stories are also different, which can be seen when comparing the vertical slopes of Façade 7 are $2.47 \%$, $3.04 \%$ and $1.81 \%$ for Story 5, 6 and 7. From resulting vertical slopes, it can conclude that the shape of the stories are not in line and each story is slightly tilted compared to the neighbour stories.

Relative deformation from the point-based method is illustrated in Fig. 7a. Large relative deformation is found in Story 5, with values varying from $-0.24 \mathrm{~m}$ to $0.41 \mathrm{~m}$ outward and inward to the towers ' central line, respectively. For other stories, the relative deformation is not more than $-0.22 \mathrm{~m}$ outward and $0.25 \mathrm{~m}$ inward. Additionally, cumulate relative deformation shows that the largest deformation of the tower is up to $0.59 \mathrm{~m}$ and $-0.64 \mathrm{~m}$ inward and outward the

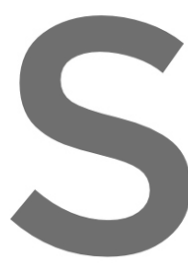
tower central line, respectively (Fig. 7b). As the deforma computed from the poin facades or the tower. cumulate relative deform 6 , other façades
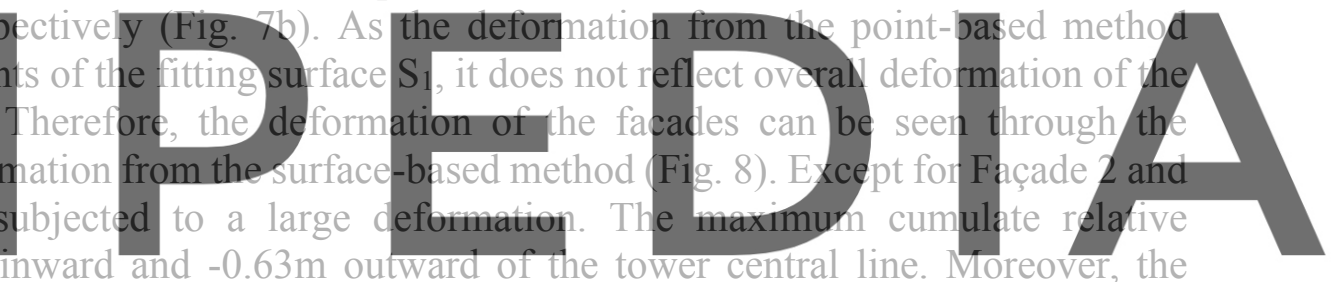
cumulate relative deformation also illustrate that deformation of the tower is not following a 


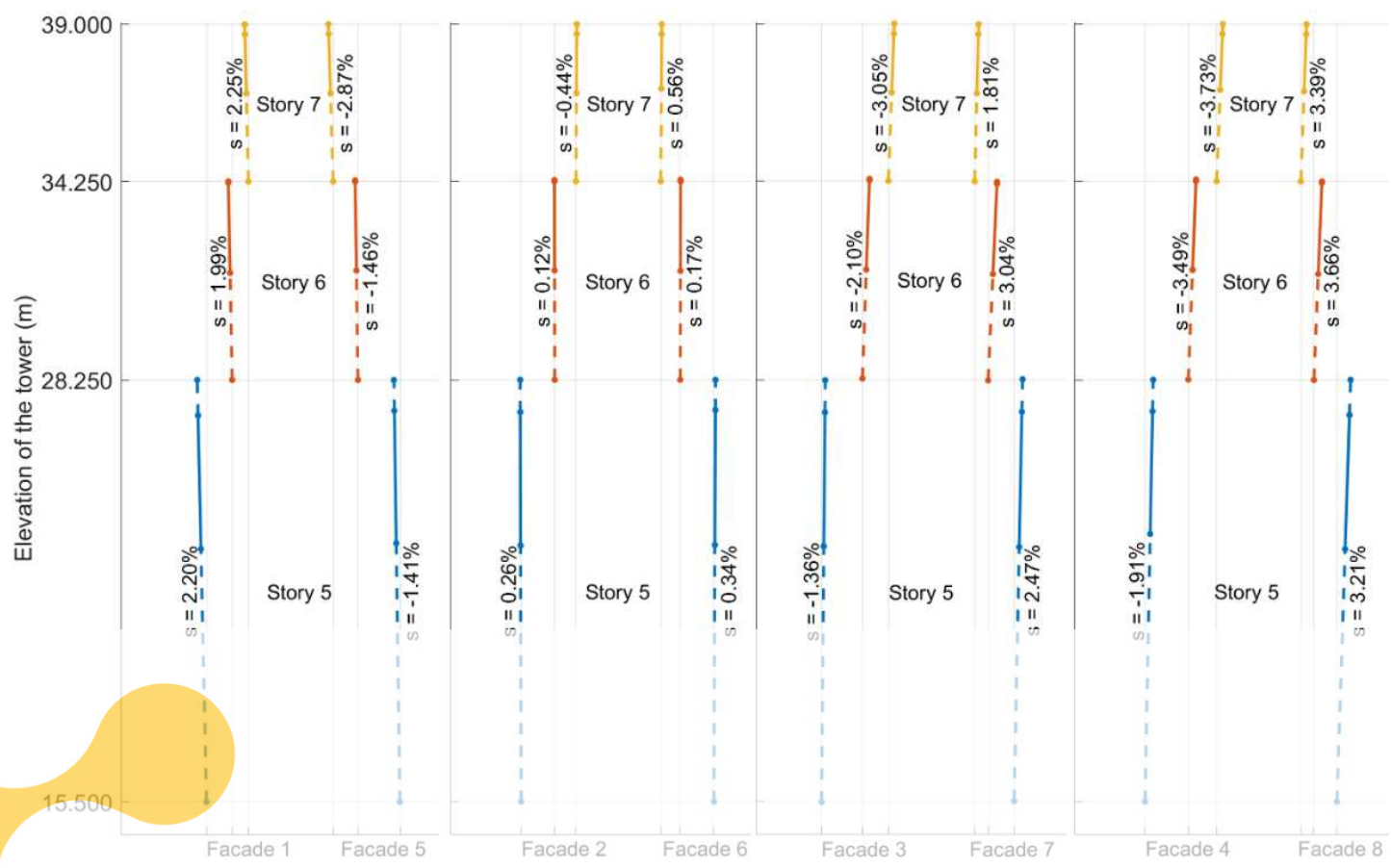

Note: (i) Negative slopes mean facades deformed inward to the tower central line and vice-versa;

(ii) Continuous lines represent the fitting surface while dashed lines are extrapolation surfaces
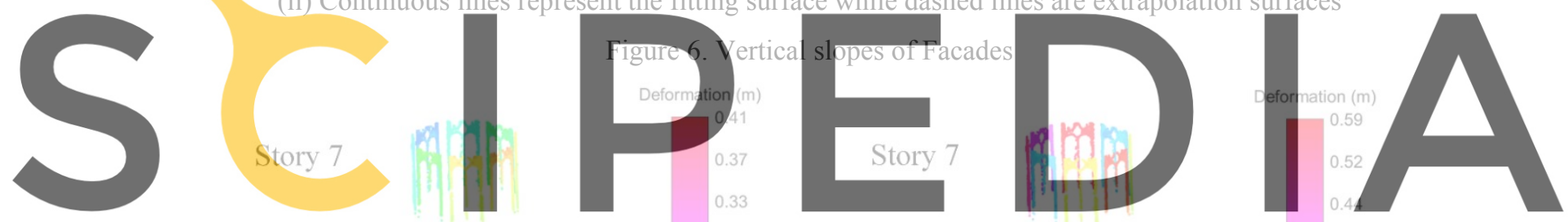

Register for free at httpsh/Nwww.scipedia.com to download the version without the watermark

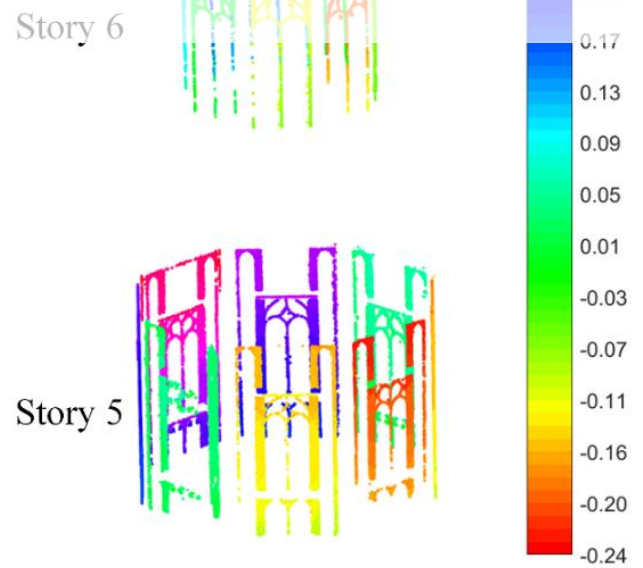

a) Relative deformation-based point

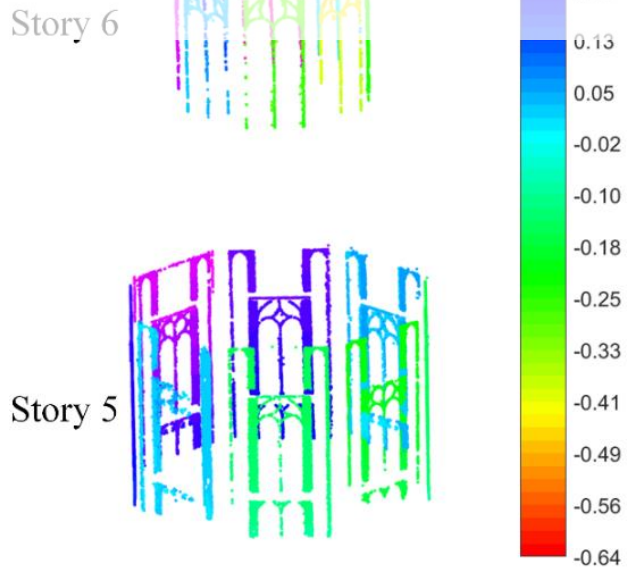

b) Accumulate relative deformation-based point

${ }^{(*)}$ Note: Negative values represent a relative deformation inward to the tower central line and vice-versa

Figure 7. Point-based relative deformation of the tower ${ }^{(*)}$ 
A visualization check shows that the tower is clearly inclined in North-West direction, which is completely in agreement with the resulted deformation estimated from the $3 \mathrm{D}$ point cloud. Moreover, the last measurement in 2011 reported that the total displacement of the tower from 1898 to 2011 is about $32 \mathrm{~cm}$. That implies the tower deformation is more than 2 times larger than that of the last record. As the tower deformation is increased significantly in the last 8 years, it would recommend to monitor the tower's deformation with higher frequency and additional technique should be implemented to measure deformations of the entire tower. However, the deformation of St. Bavo church's tower is still smaller than that of Walfridus church (Bedum, Netherlands) and San Bendetto Church (Ferrare, Germany), which total deformations are of $2610 \mathrm{~mm}$ [22] and 2530 $\mathrm{mm}$ [23], respectively.

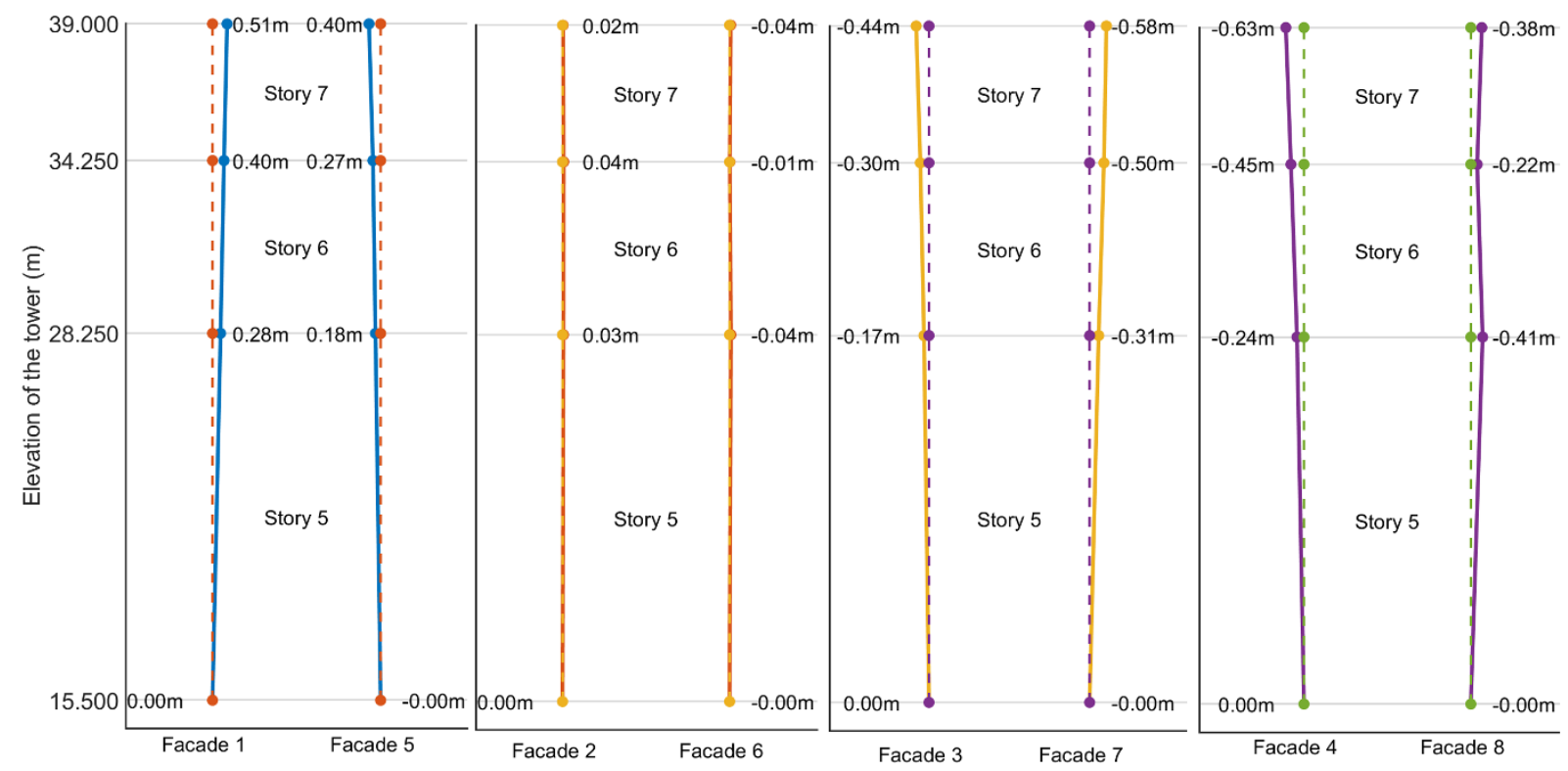

Figure 8. Cumulate relative deformation of the tower from a surface-based method

\section{CONCLUSIONS}

Heritage structures are delicate with environmental impact, which must be monitored with high frequency and accuracy, but limited budget does not always allow to implement this strategy. Using 3D Laser scanning to monitor cultural heritage structures could allow for a better understanding of the state of the structure with respect to its shape and dimensions. This is a fast and efficient technology and allows for extraction of data that can be used in structural analysis. This paper presents a methodology to measure relative deformation of a wooden tower of the St. Bavo Church in Haarlem, Netherlands. Surfaces describing the tower are extracted from point clouds of octagon facades in each story by using a RANSAC method. Next, a deformation of each surface is computed from point- and surface-based methods with the assumption that an initial or undeformed surface is perfectly vertical. The results show that the deformation of each façade in the same story and in different story are different and the large deformation is found in Story 5 with a relative deformation about $0.41 \mathrm{~m}$ outward the tower central line. Moreover, a total relative deformation at the top of Story 6 is up to $0.63 \mathrm{~m}$ outward the tower central line, which is about 2 times larger than the last record in 2011 with a total 
deformation of $0.32 \mathrm{~m}$ comparing to that in 1898 . As a cost to measure deformation of an entire tower is relatively low, a monitoring campaign can easily increase a measurement frequency to identify anomaly response of the tower. Moreover, the permanent scanning can capture a change of the tower in a specific interval time, which allows to report the deformation in nearly continuously. However, in this case, only parts of the structure within the effective range of the scanner can be measured.

\section{Acknowledgements}

This work was funded by the generous support of the European Commission through H2020 MSCA-IF, "BridgeScan: Laser Scanning for Automatic Bridge Assessment", Grant 799149. The first author is graceful for this support. The Association Friends of the Grote Kerk in Haarlem is gratefully acknowledged for their support during the Master thesis work of the $3^{\text {rd }}$ Author.

\section{REFERENCES}

[1] Truong-Hong, L., Laefer, D.F., Hinks, T., Carr, H. Combining an angle criterion with voxelization and the flying voxel method in reconstructing building models from LiDAR data. Computer-Aided Civil and Infrastructure Engineering, 28 (2012) 112-129.

[2] Teza, G., Galgaro, A., Moro, F. Contactless recognition of concrete surface damage from laser scanning and curvature computation. NDT \& E International, 42 (2009) 240-249.

[3] Gyetvai, N., Truong-Hong, L., Laefer, D.F. Laser scan-based structural assessment of wrought iron bridges: Guinness Bridge, Ireland. Proceedings of the Institution of Civil Engineers - Engineering History and Heritage, 171 (2018) 76-89.

[4] Arias, P., Riveiro, B., Armesto, J., Solla, M. Terrestrial laser scanning and non parametric methods in masonry arches inspection. in: Comission V Symposium. ISPRS, Newscalte upon Tyne, UK 2010 pp 39-44.

[5] Cabaleiro, M., Lindenbergh, R., Gard, W.F., Arias, P., van de Kuilen, J.W.G. Algorithm for automatic detection and analysis of cracks in timber beams from LiDAR data. Construction and Building Materials, 130 (2017) 41-53.

[6] Haddad, N.A. From ground surveying to 3D laser scanner: A review of techniques used for spatial documentation of historic sites. Journal of King Saud University - Engineering Sciences, 23 (2011) 109-118.

[7] González-Aguilera, D., Gómez-Lahoz, J., Muñoz-Nieto, Á., Herrero-Pascual, J. Monitoring the health of an emblematic monument from terrestrial laser scanner. Nondestructive Testing and Evaluation, 23 (2008) 301-315.

[8] Pesci, A., Casula, G., Boschi, E. Laser scanning the Garisenda and Asinelli towers in Bologna (Italy): Detailed deformation patterns of two ancient leaning buildings. Journal of Cultural Heritage, 12 (2011) 117-127.

[9] Bonali, E., Pesci, A., Casula, G., Boschi, E. Deformation of Ancient Buildings inferred by Terrestrial Laser Scanning methodology: the Cantalovo church case study (Northern Italy)*. Archaeometry, 56 (2014) 703-716.

[10] Bertacchini, E., Boni, E., Capra, A., Castagnetti, C., Dubbini, M. Terrestrial Laser Scanner for Surveying and Monitoring Middle Age Towers. in: XXIV FIG International Congress 2010- 
Facing the Challenges-Building the Capacity. FIG Federation International des Geometres, 2010 pp 1-13.

[11] Bhadrakom, B., Chaiyasarn, K. As-built 3D modeling based on structure from motion for deformation assessment of historical buildings. Int. J. Geomate, 11 (2016) 2378-2384.

[12] Muszynski, Z., Milczarek, W. Application of Terrestrial Laser Scanning to Study the Geometry of Slender Objects. IOP Conference Series: Earth and Environmental Science, 95 (2017) 042069.

[13] Dunk, T.H.v.d. Haarlem als Hollands Jeruzalem: De oorsprong van de toren van de Grote of St.-Bavokerk. Hilversum Verloren, (2016).

[14] Leica Geosystems. Leica ScanStation P40. in., 2020 pp.

[15] Leica Geosystems. Leica Cyclone 3D Point Cloud Processing Software. in., 2020 pp.

[16] Rabbani, T., Van Den Heuvel, F. Efficient hough transform for automatic detection of cylinders in point clouds. Isprs $\mathrm{Wg} \mathrm{Iii} / 3$, Iii/4, 3 (2005) 60-65.

[17] Truong-Hong, L., Laefer, D.F. Octree-based, automatic building façade generation from LiDAR data. Computer-Aided Design, 53 (2014) 46-61.

[18] Schnabel, R., Wahl, R., Klein, R. Efficient RANSAC for point-cloud shape detection. in. Wiley Online Library, pp 214-226.

[19] CloudCompare. CloudCompare. in., 2020 pp.

[20] Dillencourt, M.B., Samet, H., Tamminen, M. A general approach to connected-component labeling for arbitrary image representations. J. ACM, 39 (1992) 253-280.

[21] Laefer, D.F., Truong-Hong, L. Toward automatic generation of 3D steel structures for building information modelling. Automation in Construction, 74 (2017) 66-77.

[22] Thijssen, W. Who has the crookedest? in: de Volkshrant. Netherlands 2011 pp.

[23] Pellegrinelli, A., Furini, A., Russo, P. Earthquakes and ancient leaning towers: Geodetic monitoring of the bell tower of San Benedetto Church in Ferrara (Italy). Journal of cultural heritage, 15 (2014) 687-691. 